\title{
El Psicólogo en la Educación Infantil
}

\section{The Educational Psychologist in Pre-Primary Education}

\author{
Laura Méndez Zaballos \\ Universidad Nacional de Educación a Distancia, España
}

\author{
Isabel Yela Valdivia \\ Comunidad de Madrid, España
}

\begin{abstract}
Resumen. El objetivo de este artículo es explicar algunos de los rasgos que definen la intervención del psicólogo educativo en la etapa infantil. Con este fin organizamos la exposición en tres partes. En la primera, hacemos un recorrido histórico y legislativo por la Educación Infantil y por los Equipos de Orientación Educativa y Psicopedagógica, centrándonos en los Equipos de Atención Temprana como nicho más representativo del trabajo del psicólogo en esta etapa. En la segunda, nos preguntamos por las competencias que necesita este profesional y si poseen rasgos diferentes a las que necesitan los colegas de otras etapas educativas. La tercera y última parte desarrolla un modelo de intervención que, a nuestro juicio, confiere unos rasgos específicos al trabajo del psicólogo en la educación infantil. Este modelo está presidido por una visión holística y sistémica, basada en una práctica centrada en la colaboración y el asesoramiento. Para terminar, planteamos algunas preguntas abiertas para el debate.

Palabras clave: Psicólogo educativo, rol profesional, dilemas profesionales, educación infantil, Equipos de Atención Temprana, modelo de intervención sistémico.
\end{abstract}

\begin{abstract}
The purpose of this article is to explain some of the characteristics which define the intervention of the educational psychologist in childhood. With this aim, the presentation is organized into three parts. First, this article conducts a historical and legislative review of the Education System in childhood and through the EOEPs (Educational and Psychopedagogical Orientation Teams) focused on the EATs (Early Attention Teams), which are the most representative role of the psychologist working at this educational level. In the second part, the article presents the question of the skills which the educational psychologist needs, and if they are different to those needed by other colleagues working at other educational levels. In the third part, a model of intervention is developed which, in our opinion, deals with the specific features of the psychologist's work in early childhood education. This model uses a holistic and systemic approach, focused on collaboration and counselling. Finally, some open questions for debate are highlighted.

Keywords: educational psychologist, professional role, professional dilemmas, childhood education, Early Attention Teams, systemic model of intervention.
\end{abstract}

\section{El contexto de trabajo del psicólogo en la primera etapa educativa. Un breve recorrido histórico}

La Educación Infantil es el término con el que se denomina en España al primer nivel del sistema educativo, que abarca desde el nacimiento hasta los seis años y se divide en dos ciclos (0-3 y 3-6 años). Actualmente es un periodo educativo no obligatorio y se imparte en centros que ofertan toda la etapa educativa de enseñanza no universitaria y en centros específicos llamados Escuelas Infantiles. La gestión puede ser pública, privada concertada o privada, siendo el segundo ciclo gratuito en los centros de gestión pública (Informe Eurydice, 2010).

Esta etapa educativa tiene su origen a mediados del siglo XIX en la figura de Friedrich Fröbel, quién, en 1837, fundó la primera escuela infantil acuñando el término Kindergarten o jardín de infancia. El autor consideraba que el niño es el principal protagonista de su propia educación, que debe consistir en animar al

La correspondencia sobre este artículo debe enviarse a la primera autora al e-mail: lmendez@psi.uned.es desarrollo natural de los pequeños a través de la actividad y el juego. Seguidor de Joham H. Pestalozzi, pedagogo suizo que ponía el énfasis en la preparación del maestro, instruyó a un grupo de mujeres en su metodología para realizar su labor educadora en el Kindergarten. En España, Pablo Montesinos, seguidor de Pestalozzi e influenciado por las experiencias europeas, crea las escuelas de párvulos y diseña una formación específica para los profesores en esta etapa.

Durante el primer tercio del siglo XX se produce una expansión de centros debido al impulso de instituciones públicas y privadas y a un desarrollo pedagógico bajo la influencia de María Montesori y del Dr. Decroly. En las siguientes tres décadas (desde 1939 a 1970) el interés por la etapa fue minoritario (se produjeron algunas iniciativas privadas) y oficialmente existía una visión meramente asistencial y preparatoria de la escuela primaria. Es en la Ley General de Educación y Financiamiento de la Reforma Educativa (LGE) de 1970, donde se implanta como etapa educativa y se recoge la necesidad de favorecer el desarrollo armónico de los niños y niñas. En ella se establecían dos etapas, jardín de infancia (niños de 2 y 3 años) y la escue- 
la de párvulos (niños de 4 y 5 años). Los cambios sociales y familiares que marcaron el final del siglo XX hicieron que durante los años 80 se produjera en España un extenso desarrollo de esta etapa, tanto en relación a los índices de escolarización como en su estructura y orientación psicopedagógica.

Como consecuencia de ello, en 1985, se inicia el "Programa Experimental de Educación Infantil', que tiene una duración de tres años y el objetivo de desarrollar proyectos de innovación pedagógica adecuados a las características de la primera infancia, modificación de infraestructuras y actualización y perfeccionamiento del profesorado (Olaya, 1996). Se buscaba el cambio de paradigma de una atención basada en la asistencia, a una atención basada en la educación, con especial énfasis en las características propias del proceso de desarrollo del niño de cero a seis años y los factores que en él intervienen y que fueron descritos en el Anteproyecto de Marco Curricular para la Educación Infantil (1986). En la triangulación familia, escuela y niño, la figura del "educador" adquiere una gran importancia. Se comprende que es la persona que acompaña al niño en su descubrimiento, la que debe propiciarlo proponiendo actividades, espacios y materiales que le ayuden en el proceso de construcción del pensamiento y del aprendizaje significativo.

Finalmente, con la Ley Orgánica de Ordenación General del Sistema Educativo (LOGSE) en 1990 se impulsó definitivamente la etapa de Educación Infantil con un carácter propio y educativo. Se configura en los dos ciclos, el primero de 0-3 años, y el segundo de 3-6 años, pero con una concepción unitaria de la etapa. Se introduce el concepto de necesidades educativas especiales y contempla los mismos objetivos para los niños con deficiencias que para los niños que no las tengan, por medio de ajustes en el currículo educativo. En el año 2002 se produce una nueva reforma del sistema educativo, la Ley Orgánica de Calidad de la Educación (LOCE) que mantiene la división en dos ciclos: la Educación Preescolar (0 a 3 años) donde se vuelve a mencionar el carácter asistencial además del educativo que debe tener este período, y el segundo ciclo, Educación Infantil ( 3 a 6 años) en el que se hace una propuesta educativa clara. Introduce la enseñanza bilingüe y la materia de religión cuando los padres lo soliciten. Esta ley es derogada por Ley Orgánica de Educación LOE (2006), actualmente en vigor, la cual no divide por nombre los dos ciclos recuperando la Educación Infantil como una única etapa educativa, intentando desligarse del carácter asistencial que había impregnado el primer ciclo en la LOCE (2002).

Hasta este momento hemos mencionado la normativa de carácter estatal, pero en lo relacionado con la ordenación de la etapa, se confiere a las diferentes administraciones educativas autonomía para la regulación de los contenidos, organización y requisitos que deben cumplir los centros del primer ciclo, lo que ha provocado diferencias en el modelo de escolarización.
El segundo ciclo depende en todo el territorio nacional de las Concejalías de Educación, mientras que en el primer ciclo hay comunidades autónomas en las que todavía depende del área social. Esta disparidad se produce también en lo relacionado con el curriculum, pues mientras el segundo ciclo queda regulado por el Real Decreto 1630/2006 que establece las enseñanzas mínimas, el primer ciclo se deja en manos de las Comunidades Autónomas (CCAA) por lo que su desarrollo ha sido muy dispar. En efecto, solo seis de las diecisiete CCAA han establecido objetivos específicos para el primer ciclo (Sarmiento y Ruiz, 2011), y solo en cuatro han priorizado contenidos afectivos y del desarrollo emocional en ellos, que son la base para el crecimiento intelectual y social del niño (Brazelton y Greespan, 2005). Queremos destacar que a pesar de que el calendario de aplicación de la LOE (2006) establecía que en el curso 2008/09 todas las autonomías deberían haber concluido la legislación en la etapa infantil, se da el caso de que aún quedan cinco CCAA que solo han especificado bloques de contenido para el segundo ciclo (Informe Euridyce, 2011).

Después de este sucinto repaso histórico podemos entender que el objetivo de la etapa es el desarrollo de capacidades físicas, intelectuales, afectivas, sociales y morales de los niños. En ambos ciclos de la etapa se ha de atender progresivamente al desarrollo afectivo, al movimiento y los hábitos de control corporal, a las manifestaciones de la comunicación y del lenguaje, a las pautas elementales de convivencia y relación social, así como al descubrimiento de las características físicas y sociales del medio en el que viven. El mencionado Real Decreto 1630/2006 dispone que en el segundo ciclo las áreas sean las que siguen: Conocimiento de sí mismo y autonomía personal; Conocimiento del entorno; y Lenguajes: Comunicación y representación. Se plantea una aproximación a la lecto-escritura y a la lengua extranjera, así como iniciación de habilidades numéricas básicas, en las Tecnologías de la Información y la Comunicación (TIC) y en la expresión visual y musical. Los alumnos que así lo soliciten tendrán derecho a recibir enseñanza de la religión católica. Consideramos conveniente destacar que dicha organización debe entenderse desde una perspectiva global e integradora, evitando caer en una concepción fragmentada de la realidad.

Para ofrecer un marco unificado dentro de la Atención Temprana se elabora el Libro Blanco de Atención Temprana (G.A.T. 2000) que busca ser un documento de referencia nacional para las distintas administraciones públicas, asociaciones y familias, que permita fomentar la coordinación institucional y superar deficiencias organizativas y de recursos. En él se explicita la especial trascendencia de la etapa ya que los primeros años es donde se produce un excepcional desarrollo cerebral, el más importante de la vida (tal y como señalan los conocimientos de la neurociencia) y resultan por tanto determinantes para un desarrollo 
armonioso de los niños, por lo que destaca que la Educación Infantil tiene un marcado carácter preventivo y compensador de las carencias socio-culturales, debido a la importancia de la intervención temprana para evitar problemas de desarrollo, tanto en la población general como, y de manera especial, en los niños que puedan presentan o presenten necesidades específicas de apoyo educativo. Por lo tanto la detección de estas posibles necesidades es una de las tareas fundamentales de los tutores y se encuentra englobada en el principio de atención a la diversidad que busca la respuesta educativa más adaptada a las necesidades del alumno.

\section{La orientación Psicoeducativa: Los equipos de Atención Temprana}

Esta respuesta educativa se realiza con la colaboración de los equipos de orientación del sector o con las unidades de orientación de los centros educativos que son responsables de dar respuesta al derecho de los alumnos a recibir una orientación educativa según dispone la LOE (2006). A continuación vamos a realizar un breve recorrido por la legislación Estatal referida a estos servicios de apoyo, siguiendo lo marcado en el informe Eurydice (2011), si bien somos conscientes de la diversidad que también existe en este aspecto en las distintas CCAA (Paniagua, 2011). Destacaremos aquellas que nos aporten un valor diferenciador respecto a la labor de orientación educativa en otras etapas educativas.

Las funciones de los equipos de orientación del sector o las unidades de orientación de los centros educativos están definidas en la Orden de 9 de diciembre de 1992 por la que se regulan la estructura y funciones de los Equipos de Orientación Educativa y Psicopedagógica y en la Orden de 30 de abril de 1996 de la Dirección General de Renovación Pedagógica. Para una visión general, las hemos agrupado en tres ámbitos de actuación: Con relación a los centros, se contempla el asesoramiento y la colaboración con los equipos educativos y directivos en la elaboración de los proyectos del centro y especialmente en el plan de atención a la diversidad. Respecto a los alumnos, colaboran en la definición de las necesidades globales e individuales y proponen medidas de intervención, con especial atención a la prevención, detección y evaluación psicopedagógica de alumnos con necesidades de apoyo educativo. En este último aspecto la Orden de 14 de febrero de 1996, del Ministerio de Educación y Ciencia, por la que se regula el procedimiento para la realización de la evaluación psicopedagógica y el dictamen de escolarización y establece los criterios para la escolarización de los alumnos con necesidades educativas especiales, expresa claramente que es un proceso que debe recoger y aportar información relevante del y para el alumno y su contexto familiar y escolar.
El proceso de evaluación es entendido como interdisciplinar, que trasciende los límites del equipo de orientación psicopedagógica y que debe incorporar a los profesionales que participan directamente en los procesos de enseñanza-aprendizaje. En todo caso, la interpretación de la información obtenida debe ser coherente con la concepción interactiva y contextual del desarrollo y del aprendizaje (Retortillo y Puertas, 2004).Y finalmente, en cuanto a la familia, ofrecen asesoramiento relacionado con la prevención, detección e intervención en las posibles dificultades del alumnado. Aspecto que retomaremos un poco más adelante.

Todos los Equipos de Orientación tienen una composición interdisciplinar. Están formados, en su mayoría, por psicólogos, pedagogos o psicopedagogos y trabajadores sociales, y están distribuidos por sectores geográficos, por lo que son itinerantes. Los Equipos encargados de la orientación en la etapa de Educación Infantil son los denominados en el artículo $4^{\circ}$ de la Orden de 9 de diciembre de 1992 como Equipos de Atención Temprana cuya composición, además pueden contar, con maestros de Pedagogía Terapéutica y de Audición y Lenguaje. De tal forma que a la Educación Infantil se le adscriben la atención de unos equipos especializados respetando y promocionando de esa forma la entidad de la etapa y por lo tanto de los equipos que van a realizar la orientación educativa en la misma.

Esta especificidad es desarrollada posteriormente en la Orden de 30 de abril de 1996 de la Dirección General de Renovación Pedagógica, que señala como principal objetivo de los Equipos de Atención Temprana la contribución a la mejora de las condiciones educativas de los centros que escolaricen a los niños y niñas con necesidades educativas especiales, bien sea por condiciones personales de discapacidad o situaciones sociales desfavorecidas. Con lo que se destaca, una vez más, la relevancia de la prevención y compensación de desigualdades, ya que es justamente en este periodo educativo donde se ponen de manifiesto gran parte de las dificultades que afectan a la población escolar. Los principios de integración y normalización deben regir las actuaciones de los Equipos de Atención Temprana, quienes tienen que proporcionar la atención educativa a las necesidades educativas especiales tan pronto sean detectadas y evaluadas, dando respuesta a las necesidades de los niños y niñas y a la de sus familias.

El apoyo a las familias es esencial en esta etapa educativa, son los Equipos de Atención Temprana los que tienen que promover la relación entre la familia y escuela, la información entre estos dos contextos debe ser fluida si se quiere dar una educación coherente a los niños y niñas. Se debe velar por el adecuado desarrollo de los procesos de acogida e inserción de los alumnos con desventaja. Asimismo, en los procesos de escolarización de los niños y niñas con necesidades educativas especiales los padres deben tener una infor- 
mación clara y fidedigna, que les permita comprender en qué consisten las medidas educativas propuestas, que éstas tienen un carácter temporal y que deben ser revisadas, orientado en aquellos aspectos que resulten básicos para atender adecuadamente a los niños y niñas con necesidades educativas especiales o en situación de desventaja, colaborando en el establecimiento y desarrollo de programas formativos. En no pocas ocasiones los Equipos de Atención Temprana comparten con otros servicios de atención a la infancia, la responsabilidad de comunicar la "primera noticia", debiendo prestar especial atención al momento, modo y forma de comunicación (G.A.T. 2004). En general, el trabajo con las familias debe perseguir la participación activa de los padres y de las madres, buscando generar una especial sensibilidad educativa en la familia.

Para finalizar el recorrido por la funciones asignadas a los Equipos de Atención Temprana, quisiéramos exponer cómo en la mencionada Orden del 30 de abril, al hacer referencia a las funciones de apoyo especializado, especifica la función de intervenir directamente con el alumno con necesidades especificas de apoyo educativo, ya sea de manera individual o en pequeño grupo, en el aula o fuera de ella, también para los psicólogos y pedagogos. En este tipo de actuaciones es difícil deslindar los aspectos propiamente educativos de los terapéuticos o sociales, por ello existen amplias zonas de coincidencia de en las responsabilidades con los servicios de salud y servicios sociales, lo que obliga a realizar una estrecha colaboración y una deseable convergencia entre todas ellas. La necesidad que se desprende es la de un modelo global de intervención desde las perspectivas educativa, social y de la salud, que tal y como propone el Libro Blanco de la Atención Temprana (G.A.T. 2000), esté encaminado a proporcionar una atención educativa global, sistematizada y adecuada a la población infantil de 0 a 6 años.

A la vista de estas funciones y de su complejidad e importancia para el desarrollo infantil temprano, nos preguntamos qué habilidades y competencias tiene que poseer el psicólogo que trabaja en esta primera atapa educativa.

\section{Competencias del psicólogo en la Educación Infantil}

Las funciones que acabamos de señalar en el apartado anterior, necesitan de un psicólogo educativo que fundamente su trabajo en los conocimientos científicos de la psicología, tanto generales como específicos, estos últimos centrados en los procesos de desarrollo y aprendizaje de los menores de 6 años. Por otra parte, la concreción de estas funciones se caracteriza por estar dirigida a una población diversa de niños y por llevarse a cabo en un escenario educativo concreto, donde, como hemos comentado antes, resaltan los aspectos relacionales, compensatorios y preventivos.
Partiendo de esta realidad podemos preguntarnos qué competencias necesita el psicólogo para realizar su trabajo en este contexto y para esta población y si son diferentes a las que necesitan otros colegas que ejercen su trabajo en otra etapa educativa. Para contestar a esta pregunta vamos a seguir el modelo propuesto por la Asociación Nacional de Psicólogos Escolares de EE UU (Méndez y Escudero, 2011; NASP, 2006, 2010), ya que a nuestro juicio reúne elementos especialmente significativos para la intervención psicológica en la educación infantil.

Esta asociación propone ocho competencias, cuatro de carácter general y cuatro específicas. Comenzaremos por las primeras. Las competencias generales o básicas están relacionadas con los rasgos éticos y legales de la profesión, con las habilidades sociales y colaborativas, con el uso de la tecnología y con la capacidad para trabajar con diversidad de necesidades y servicios. Para algunos autores (Ramey, Ramey y Gaines, 2004; Reschly y Ysselyke, 2002), aunque estas habilidades forman parte del catalogo de competencias de cualquier psicólogo, algunas de ellas son especialmente relevantes para el psicólogo educativo que interviene en la etapa educativa infantil. De entre todas destacamos dos de ellas.

Habilidades interpersonales y colaborativas. En esta etapa, tal y como hemos mencionado, es ampliamente aceptada la importancia de establecer una buena relación interpersonal con los educadores y las familias. Para llevar a cabo su trabajo de forma satisfactoria el psicólogo tiene que entender la importancia de trabajar con ambos de forma colaborativa y comprender el sentido sistémico de las respuestas que cada uno de ellos dan a un mismo problema. Desde esta perspectiva, una de sus misiones más importantes es trabajar para reducir las divisiones o contradicciones que puedan surgir y crear un ambiente adecuado para la negociación y el consenso, estimulando la implicación de todos en la resolución del problema. Es precisamente la capacidad para desarrollar un asesoramiento colaborativo una de las habilidades más necesarias para el psicólogo que trabaja en la educación infantil (Rosenfield, Levinsohn-Klyap, y Cramer, 2010). A este punto volveremos en apartados posteriores.

Dentro de las habilidades interpersonales, queremos resaltar un aspecto considerado muy relevante para las asociaciones profesionales (APA, 2006, NASP, 2010) y académicas (Rosenfield, Levinsohn-Klyap, y Cramer, 2010). Nos referimos a la necesidad de que los psicólogos desarrollen habilidades multiculturales para intervenir en contextos cada vez más diversos. Como se ha visto, la Educación Infantil tiene un claro componente compensador, para muchas familias de culturas minoritarias supone el primer contacto con una institución formal en el país de acogida. Un psicólogo sensible a esta realidad tendrá en cuenta el origen cultural de las familias, considerándolo un elemento significativo para explicar e interpretar los comportamientos y 
actitudes paternas respecto a la escuela o a los hábitos de crianza.

Responsabilidad legal y ética. Hemos querido resaltar esta competencia porque creemos que es la gran olvidada en la formación de los psicólogos (Del Río, 2009). El respeto al código deontológico y el comportamiento responsable es esencial para una buena práctica cuando el profesional interviene con menores que, por su momento evolutivo, no pueden manifestar sus deseos o desacuerdos. Además de los estándares de comportamiento ético en el trabajo con menores, queremos resaltar la necesidad de que los psicólogos reconozcan la importancia de una formación continua para preservar la calidad de sus actuaciones y la actualización de sus competencias. Esta última cuestión es interesante si pensamos en la carencia de formación específica que en nuestras universidades se ofrece a los psicólogos para especializarse en la intervención temprana. Por un lado, tenemos un Grado de Psicología generalista, donde el espacio para la formación más especializada es muy reducido o prácticamente inexistente. Por otro, la especialidad en orientación educativa contemplada en el máster universitario de formación del profesorado de educación secundaria obligatoria, debido en buena parte a su escaso número de créditos, no puede capacitar para la función profesional especializada en un campo concreto de la orientación, como es la intervención temprana.

Por el momento hemos comentado las competencias básicas que deben estar presentes en la intervención psicológica temprana, explicando dos de las más significativas. Ahora vamos a referirnos a aquellas más específicas. En la propuesta que estamos presentando se señalan 4 competencias, dos de las cuales se centran en potenciar en los estudiantes el desarrollo de habilidades académicas, su bienestar mental y la adquisición de competencias sociales y para la vida. Las otras dos se refieren, por un lado, a la capacidad del psicólogo para utilizar un sistema de decisiones riguroso y un método adecuado para evaluarlo, y por otra, a la competencia para desarrollar servicios psicológicos desde una visión sistémica. Vamos a comentar dos de ellas marcando las implicaciones que tienen para los profesionales que trabajan en la etapa infantil.

Facilitar las habilidades sociales, la salud mental y las competencias para la vida. Debido al momento del desarrollo de los niños, en la primera etapa educativa es quizá todavía más importante que en otras etapas abandonar una visión parcial para adoptar en la intervención una mirada holística de la persona. Esto supone que además de contribuir al desarrollo de las habilidades cognitivas y de aprendizaje, centradas fundamentalmente en la adquisición de las bases para los aprendizajes posteriores, el psicólogo también se ocupará de que los niños comiencen a adquirir las habilidades para un desarrollo posterior sano y positivo. Para ello tendrá que superar una tradición en la psicología escolar que sitúa el foco de la intervención en las nece- sidades educativas especiales y las dificultades de aprendizaje, para resituarlo en la detección de aquellos problemas que puedan derivar en trastornos de personalidad o sociales. Consecuentemente ayudará a implementar programas preventivos que favorezcan la salud, incidiendo fundamentalmente en aquellos que potencian las relaciones socioafectivas y las competencias de adaptación. Para muchos niños la escuela es el primer entorno en el que se empiezan a gestar sus relaciones con los iguales y donde tienen sus primeras experiencias de adaptación a un nuevo contexto diferente de la familia. Por ello los aspectos afectivos son especialmente significativos en esta etapa, tal y como se ha señalado previamente.

Desarrollo de servicios desde una perspectiva sistémica. Este dominio de competencia tiene que ver con una toma de postura y una apuesta clara por un modelo de intervención de carácter sistémico. Esto significa trascender una visión de los problemas centrados en cada uno de los elementos, para pasar a tener en cuenta las interrelaciones entre ellos. Desde esta perspectiva, el foco de la intervención psicológica no estará en el nivel individual y en los problemas de aprendizaje del niño sino en todos los sistemas en los que se genera el problema y su solución, como la familia, la escuela, la comunidad, o la política educativa. Este posicionamiento tiene sus raíces en la psicología ecológica donde todos los contextos donde el niño se mueve y que afectan directa o indirectamente a sus comportamientos, deben considerarse como sistemas con múltiples elementos que inciden en el aprendizaje y desarrollo de este alumno de muy diversas formas. Aceptar este planteamiento, supone que el psicólogo que trabaja en esta etapa educativa tiene que estar preparado no solo para intervenir con el niño y su problema sino que debe ser competente para intervenir en los otros contextos sociales e institucionales, analizando de qué forma se afectan mutuamente y cómo afecta a las prácticas de aprendizaje. Lo que implica dejar de ver al psicólogo como un solucionador de problemas para poner en valor su papel mediador como enlace o puente entre los diferentes niveles, con especial atención a la familia y a la escuela, contribuyendo a que cada uno entienda y valore el papel del otro.

Toma de decisiones fundadas y responsables. Un profesional de la psicología competente debe: a) conocer los elementos relevantes de un problema, posicionándose, como ya hemos señalado, en una visión holística y multifactorial del problema, b) saber tomar las decisiones adecuadas de una forma colaborativa y teniendo en cuenta las aportaciones de otros agentes o profesionales, c) conocer las técnicas y métodos para evaluar las actuaciones y también ayudar a otros, educadores o familia, a valorar la situación, d) actuar en consecuencia, haciendo partícipes a los que de forma directa o indirecta puede estar inmersos y evaluar conjuntamente con ellos los resultados. Por lo tanto, debe formarse en el manejo de las técnicas de análisis y eva- 
luación más adecuadas, de entre ellas dos son a nuestro juicio las más relevantes en la etapa infantil. Nos referimos a la observación y a la entrevista y la forma de adaptar ambas técnicas a los diferentes agentes con los que trabaja, padres, profesores y niños.

En este apartado se ha intentado responder a la pregunta planteada al comienzo del mismo, comentando aquellas competencias que necesita el psicólogo en la primera etapa educativa. En el apartado que comienza ahora vamos a responder a otra cuestión muy relacionada con la anterior: ¿cómo ejerce el psicólogo su papel?, ¿posee rasgos distintos al rol que asume en otras etapas educativas?

\section{El papel del psicólogo educativo. Propuesta de un modelo de intervención}

Al igual que dijimos al referirnos a las competencias, la forma en que el psicólogo ejercer su rol comparte características con el adoptado en otras etapas educativas. Sin embargo, también ahora, podemos señalar un rasgo que, aunque no de forma exclusiva, sí adquiere características particulares cuando el psicólogo educativo trabaja en la Educación Infantil. Nos referimos al modelo desde el que ejerce su trabajo. Como ya hemos dicho antes, la intervención de este profesional debe estar presidida por una visión holística y sistémica, lo que nos lleva a defender una práctica basada en la colaboración y el asesoramiento.

No es este modelo el seguido tradicionalmente por los psicólogos para ejercer su trabajo. Por el contrario, su papel ha estado basado en la evaluación utilizando pruebas estandarizadas y en la intervención directa, como así lo demuestra la percepción que los profesores de diferentes países europeos tienen de su papel (Farrell, Jimerson, Kalambouka, y Benoit, 2005). Sin embargo las tendencias de la psicología en las últimas décadas, defendidas por asociaciones de la psicología y por los propios usuarios (Farrell, 2009, Méndez, 2011) abogan por trascender este modelo en defensa de un modelo de consulta o asesoramiento en cualquiera de las etapas educativas incluida la infantil (Hojnoski y Missall, 2006; Wesley y Buysse, 2004). Desde esta perspectiva, el psicólogo trabajará sobre todo, con una concepción de servicio indirecto cuyo foco principal está en el trabajo colaborativo con profesores y familias para contribuir no solo a resolver problemas sino también a evitarlos desde un planteamiento claramente preventivo. Esta tarea de asesoramiento puede variar desde el análisis de necesidades, a proporcionar consejo o facilitar recursos y formación (Nieto y Portela, 2006).

A pesar de este cambio de tendencia, en las diferentes propuestas nacionales e internacionales las prácticas que desarrollan los psicólogos, también los que trabajan en la etapa infantil, están todavía lejos de centrarse en tareas de asesoramiento (Gine, Gracia,
Vilaseca y Balcells, 2009). A continuación señalamos dos cuestiones que esperamos ofrezcan algunas claves para definir el papel del psicólogo educativo infantil cuando interviene desde este modelo.

\section{A quién dirige su intervención}

Uno de los problemas con los que se encuentra el psicólogo es la multiplicidad de agentes que pueden solicitar su ayuda o asesoramiento. En infantil es característico que las demandas le lleguen por múltiples vías, profesores, familias, equipo directivo o instituciones externas y que éstas lleguen de forma individual o colectiva. Indudablemente esta pluralidad de agentes y modalidades requiere del profesional una preparación muy amplia y completa en técnicas de asesoramiento. Las diferencias entre los grupos, por ejemplo profesores y equipos directivos, y las que pueden existir dentro del mismo colectivo, pensemos en la realidad cada vez más diversa de familias, requiere un psicólogo que sepa trabajar en contextos cada vez más plurales. No es lo mismo orientar a un grupo de educadores que a un profesor concreto o asesorar a una sola familia que hacerlo en una escuela de padres. Cada uno de estos casos requiere técnicas y habilidades específicas, como por ejemplo conocer los procedimientos más efectivos para comunicar información a audiencias diversas (padres y profesores).

Por otra parte, es frecuente que los diferentes agentes requieran su ayuda ante un mismo problema. En este caso el psicólogo tendrá, como ya hemos comentado al hablar de las competencias requeridas, hacer de mediador y facilitar a todas las partes una visión comprehensiva del problema o situación, lo que supone facilitar canales de comunicación y colaboración entre ellos, tarea no siempre fácil. Para algunos expertos como Dunst (2004) una de las funciones del profesional es ayudar a las familias a mejorar o ajustar sus pautas educativas, intentando que el análisis de la situación y las estrategias de intervención sean compartidas por los educadores de sus hijos.

Unido a estas cuestiones y relacionado también con los asesorados, uno de los aspectos que más influye en el rol del psicólogo desde este modelo es la conceptualización y creencias que tienen padres, profesores, equipos directivos o administración educativa sobre el objeto de sus demandas. Para que el proceso de evaluación e intervención funcione es fundamental considerar las creencias, expectativas e historia familiar (Gine et al., 2009). Es importante que el psicólogo conozca qué piensan por ejemplo los padres sobre aspectos generales como el desarrollo infantil, el papel de la escuela en esta primera etapa o sobre su propio papel en el proceso educativo de sus hijos.

De la misma forma, conocer las ideas previas, teorías implícitas y creencias de los educadores sobre el desarrollo del niño y sus problemas resulta especial- 
mente relevante. Es importante que el psicólogo conozca la actitud del educador cuando plantea la demanda. Una actitud, más o menos positiva, influirá en la forma de percibir el papel del psicólogo y su propio papel como educador en la solución del problema planteado. También es importante que el profesional conozca la actitud que el educador tiene respecto a la familia, que sepa si los educadores tienen confianza en las pautas de crianza de los padres, ya que confiar en ellos promoverá que se sientan respaldados y recuperen la confianza en sí mismos (Law, Hanna, King, Hurley, King, y Kertoy, 2003).

Relacionado con esto, para algunos especialistas en el asesoramiento (Wesley y Buysse, 2004) una de sus bases es trabajar con los asesorados, en nuestro caso con los educadores y profesores, para aclarar qué piensan sobre el mismo proceso de asesoramiento, sobre cuáles son sus fines, o qué elementos caracterizan el rol del asesor. Junto a esto también necesitan conocer qué aspectos según su criterio definen el problema o situación objeto de la demanda y qué elementos pueden estar influyendo en la toma de decisiones.

\section{Cómo interviene}

Desde el modelo que estamos defendiendo en este artículo, el trabajo del psicólogo en la etapa infantil se aleja de planteamientos centrados en los problemas y dificultades del niño para poner el foco de atención en el apoyo a todos los elementos que definen el sistema, familiar y escolar, donde aprende y se desarrolla.

¿Qué supone aceptar esta idea? Para explicar sus repercusiones, retomamos el trabajo con las familias como un elemento característico de la intervención en esta etapa educativa. La importancia otorgada a este tema y la forma de abordarlo es un rasgo claramente diferenciador. Si analizamos el trabajo que el psicólogo realiza con las familias en primaria o secundaria, observamos que se centra en estimular y potenciar las relaciones familia-escuela desde la evidencia de que una colaboración estrecha entre ambos entornos beneficia la adquisición de habilidades académicas y sociales en los estudiantes (Cox, 2005).

Sin embargo, el psicólogo que trabaja en la primera etapa educativa tiene que ir más allá de este planteamiento y situar el trabajo con la familia en el centro de su intervención, considerando el apoyo al entorno familiar, no solo una forma indirecta de influir en el aprendizaje del niño, sino una meta en sí misma. Desde este posicionamiento, no solo apoyará el diseño de entornos de crianza efectivos en el entorno familiar sino que, y de forma muy importante, apoyará y guiará a la familia en su rol de cuidadores y en la forma en que estructuran las relaciones y los hábitos de crianza. Exponemos brevemente dos ideas (Gine et al., 2009) que el psicólogo debe admitir cuando trabaja con las familias desde este modelo, asumiendo la colaboración con los padres como un proyecto imprescindible para la mejora en la intervención educativa temprana.

La primera idea tiene que ver con la necesidad de que el profesional de la psicología no se deje llevar por una visión patologizada del niño y de su familia. Conseguir esto es todo un reto cuando impulsado, en buena medida, por las prioridades que marca la administración educativa, su trabajo diario se define fundamentalmente por tareas de diagnóstico e intervención en la discapacidad o los trastornos del desarrollo. A pesar de ello, el psicólogo tiene que hacer un esfuerzo en poner su mirada no en las carencias o debilidades de las familias sino en sus fortalezas. Su principal objetivo será conseguir una mayor capacitación de todos sus miembros que les ayude a tener un afrontamiento positivo (Pozo, Sarría y Méndez, 2006). Para conseguirlo mostrará interés por el bienestar general de la familia y sus necesidades, por todo aquello que les preocupa y no solo por los problemas relacionados con su hijo.

La otra idea importante, es que el psicólogo debe impulsar la participación de los padres en el diagnóstico del problema y en su solución, animándolos a manifestar sus intereses y deseos y teniendo en cuenta su opinión y preferencias al plantear los objetivos de la intervención. Como decíamos en otro apartado, el psicólogo abandona su papel de experto para compartir con los padres los objetivos y resultados de la intervención, lo que supone tomar decisiones conjuntamente como principio básico del trabajo en colaboración (Blue-Banning, Summers, Frankland, Nelson, y Beegle, 2004).

Hasta aquí hemos hablado de la intervención con las familias, ahora nos referimos al trabajo que el psicólogo lleva a cabo con los docentes. Sin duda los aspectos a considerar son muchos y han sido tratados en la literatura reciente (Paniagua, 2011). En este artículo limitamos la exposición a explicar algunos elementos que definen la interacción que se establece entre psicólogos y educadores y las características del trabajo conjunto que realizan.

Con este fin seguimos el trabajo de Weley y Buysse (2004) en el que se aborda las diferentes fases del asesoramiento, señalando en cada una de ellas aspectos que nos ayudan a explicar la relación que establecen psicólogos y educadores. A continuación exponemos brevemente los que nos parecen más relevantes.

En la fase inicial, uno de los elementos que parecen condicionar más esta relación es la experiencia previa de ambos, tanto referida a la etapa educativa como a los trastornos del desarrollo más frecuentes en el periodo evolutivo temprano. Además, hay que añadir, un aspecto frecuentemente olvidado, la experiencia previa que educadores y psicólogos han tenido sobre el asesoramiento colaborativo. El haber tenido experiencias diferentes o el hecho de que carezcan de ella, puede provocar la aparición de discrepancias y la presencia de expectativas distintas sobre los fines y desarrollo del proceso de colaboración, un ejemplo representati- 
vo es cómo la mayoría de los educadores esperan que los psicólogos centren su trabajo de forma exclusiva en los alumnos con trastornos del desarrollo o necesidades educativas especiales. Para paliar estas dificultades, es imprescindible que pacten los roles y las metas $\mathrm{y}$ analicen juntos qué conocimiento puede aportar cada uno de ellos al proceso, para lo que es fundamental la generación, desde esta fase inicial, de una sólida confianza mutua.

En la fase de identificación del objeto de la intervención, lo más importante es la puesta en común de aquellos aspectos que ayudan a valorarlo e interpretarlo. Es frecuente que educadores y psicólogos discrepen en la selección de los elementos sobre los que hay que intervenir y en el peso que otorgan a cada uno de ellos. Estas diferencias, como comentamos en el punto anterior, están influidas por el tipo de experiencias que ambos poseen. Puede que el psicólogo tenga escasa experiencia en esta etapa educativa, en su desarrollo curricular, o en las técnicas de intervención en el aula, mientras que el educador puede carecer de conocimientos sobre los niños con algún tipo de trastorno en su desarrollo y las necesidades educativas y psicológicas que se presentan. Estas diferencias en habilidades, conocimiento y experiencia puede condicionar todo el proceso de colaboración. Por ello es imprescindible crear espacios de discusión y puesta en común sobre aquello que se quiere cambiar o mejorar y los elementos que pueden influir en este cambio.

La siguiente fase tiene que ver con la elección de estrategias de intervención. En un primer momento, el psicólogo, a partir de sus conocimientos especializados, ayudará a los educadores en el análisis de las estrategias que ya han implementado, valorando conjuntamente su efectividad. En un segundo momento, y partiendo del análisis anterior, el psicólogo debe poner sus habilidades y conocimientos al servicio de los educadores, posibilitando un acuerdo y consenso sobre qué nuevas técnicas se deben activar. Su conocimiento especializado sobre una amplia gama de estrategias centradas en el alumno, en el grupo, o en episodios críticos del proceso de enseñanza, sin duda beneficiará el acuerdo, ya que podrá ayudar a los educadores a seleccionar aquellas que pueden resultar más útiles y efectivas para ellos y, lo que es más importante, con las que se sientan más cómodos y seguros. Sin olvidarse de anticipar las dificultades que pueden surgir en el proceso de y discutir conjuntamente la forma de evitarlas.

La puesta en marcha de las estrategias escogidas, es la última y más crucial en el proceso. Durante la misma, el rol del psicólogo variará dependiendo, en parte, de las habilidades y conocimientos que posean los educadores. En cualquier caso el primer rol que asumirá será el de observador sobre el terreno de las estrategias implementadas. Una vez hecho esto, su papel cambiará pasando del rol de modelador (podemos hacerlo así...) a un rol de apoyo o "coach" (está muy bien así, mañana daremos el siguiente paso...).
Un aspecto que, sin duda, está condicionando las relaciones y distribución de papeles en esta fase, es la ausencia del psicólogo en la mayor parte del tiempo que dura la intervención. Por necesidades de su propio trabajo y la falta de recursos del propio sistema educativo, los psicólogos, como hemos visto en la primera parte de este artículo, acuden a los centros de forma itinerante, lo que les impide tener una presencia continuada en los mismos. Esto implica que los educadores se ven forzados a implementar el plan de acción en solitario. Para paliar en parte este problema, los psicólogos deben evitar que los educadores se sientan solos y "abandonados", instaurando mecanismos de comunicación más allá de la presencia física. Por otra parte, les deben proporcionar métodos y herramientas para que puedan monitorizar y documentar el proceso para poder evaluarlo luego conjuntamente.

\section{Algunas preguntas a modo de conclusión}

Terminamos este artículo planteando tres cuestiones que vertebran el presente trabajo y dejan abiertas vías de discusión para el debate.

¿Educación preescolar o educación infantil?, aunque sin duda es una pregunta ya antigua, los diferentes cambios legislativos, que hemos visto al principio de estas páginas, muestran que aún está viva para el debate. A lo largo del artículo hemos resaltado la relevancia de la etapa de educación infantil, preventiva y compensadora, planteando la necesidad de que haya una apuesta clara por parte de la administración que garantice una atención de calidad desde el nacimiento. Para cumplir este objetivo se debe potenciar una buena formación especializada para todos aquellos servicios que trabajen en esta etapa, de forma especial para psicólogo educativo.

¿Una formación generalista o específica?, la respuesta a esta cuestión esta clara para nosotros. El psicólogo educativo necesita desarrollar unas competencias y habilidades, que aun compartidas con colegas de otras etapas educativas, poseen unos rasgos diferentes. Aceptando esta idea, las más significativas son las relacionadas con las habilidades para trabajar de forma colaborativa con educadores y familias desde un planteamiento ético, destacando la importancia de adoptar una mirada holística de la persona que potencie la puesta en marcha de programas preventivos que favorezcan la salud e incidiendo en las relaciones socioafectivas y las competencias de adaptación en los niños. Todo esto supone la formación de un profesional capaz de tomar decisiones fundadas, responsables y compartidas con otros agentes educativos.

¿Prevenir o remediar?, el último interrogante que planteamos tampoco es nuevo, pero sigue estado presente en el debate entre los profesionales y los usuarios de sus servicios. Al comienzo de este artículo hemos visto cómo el marco legislativo prioriza las funciones 
relacionadas con la atención a los niños de necesidades educativas especiales, lo que ha llevado a adoptar una forma de intervenir centrada en el niño y con frecuencia al margen de los contextos donde se desarrolla, como la escuela o la familia. Alejándonos de este planteamiento, hemos querido ofrecer otra alternativa de intervención que asume una visión preventiva, holística y sistémica, basada en una práctica centrada en el asesoramiento y la colaboración con educadores y familia. Esta forma de entender la intervención, no excluye prácticas como la evaluación o la intervención individual, pero sí supone una nueva forma de entenderlas, más ecológica y contextual. Esta aproximación no está exenta de dilemas para el psicólogo educativo que trabaja desde el modelo. Uno de los más relevantes ha sido formulado por Norwich, (2005) en un trabajo muy interesante sobre los dilemas identitarios de la profesión. Para este autor cuanto más se aleje su intervención del niño para poner el foco en los diferentes contextos que tienen que ver con el desarrollo infantil, más se desdibujará su papel, al coincidir con las funciones de otros profesionales (profesores, pedagogos, o educadores sociales) y con otras áreas de la psicología, como la clínica. La respuesta, como nos indica el propio autor, puede estar en adoptar sin miedo los conocimientos y perspectivas de otras disciplinas, al tiempo que contribuimos a hacer presente el conocimiento psicológico en las prácticas de los diferentes profesionales, no psicólogos, que intervienen en esta etapa.

Estas cuestiones y otras muchas están aún abiertas para la discusión. Esperamos que la lectura de estas páginas haya aportado algunas respuestas y abierto nuevos interrogantes para el debate.

\section{Extended Summary}

This article deals with the identity of educational psychologists working in pre-primary education and consists of three parts. The first part provides a brief history of the development of pre-primary education and the relevant legislation in Spain. Pre-primary education in this country is currently divided into two cycles (0-3 and 3-6 years of age), is designed to prevent and compensate for socio-cultural inequalities, and has the aim of developing children's physical, intellectual, affective, social and moral capabilities. Based on this analysis, we show how the characteristics of this stage imply the need for specialised consultation. This has led to the setting up of specific interdisciplinary teams providing educational and psychoeducational guidance, known as Early Care Teams, distributed by geographical sectors, based on an external intervention model of educational consultation. The work of these teams has three focuses: the school, the child and the family, with attention to the demands of the schools and direct intervention with pupils with specific educational support needs clearly predominating.

In the second part, we discuss the skills psychologists need to do their job in this setting and for this population, and whether they are different from those needed by psychologists working at a different educational stage. In answering this question we follow the model proposed by the National Association of School Psychologists in the United States, which posits eight skills - four general and four specific. Of the former, we regard interpersonal and collaborative skills as the most significant, as they are the basis for providing collaborative consultation. One particularly important factor is the need for educational psychologists to develop multicultural skills in order to intervene in increasingly diverse settings. We also consider skills in the domain of legal and ethical responsibility to be essential for good practice when professionals intervene with minors who, because of their developmental stage, are unable to manifest their wishes or disagreements.

In respect of specific skills, we discuss two further competencies: enhancing social skills, mental health and life competencies and data-based decision-making and accountability. In our opinion, it is even more important when intervening in the initial stage of education to adopt a holistic view of the person. We therefore propose system-based service delivery, in which the focus of the psychological intervention is not always at the individual level and on the child's learning problems, but has to do with all the systems in which the problem and its solution are generated, such as the family, the school, the community or the educational policy. Accepting this approach means that psychologists working at this educational stage must be equipped not just to intervene with the child and his or her problem, but must also be competent to intervene in the other social and institutional settings and develop their mediating role as a link or bridge between the different levels, paying special attention to the family and the school. They must also be able to ascertain the major elements of a problem from a holistic and multi-sector viewpoint, take the appropriate decisions collaboratively, know the relevant assessment techniques and methods, and act in consequence, making those who are directly or indirectly involved a party to what they do and evaluating the outcomes jointly with them.

The final part attempts to answer another question closely linked to the previous one: how do psychologists perform their role and does this role have different features from those performed at other educational stages? We highlight the importance of the intervention model and propose a practice based on collabora- 
tion and counselling, and a holistic and systemic approach. According to this perspective, the psychologist's job will consist primarily in providing an indirect service whose main focus is on collaborative work with teachers and families, and aimed at helping not only to treat problems, but also to prevent them by using a distinctly preventive approach. We highlight two aspects we regard as crucial in defining the role of pre-primary educational psychologists:

Who their intervention is aimed at. The differences between the different groups -for example teachers and the senior management team-and the differences that may exist within the same group, require psychologists who are able to work in increasingly plural settings. With regard to the people for whom consultation is provided, one of the factors with the biggest influence on the psychologists' role in this model are the conceptualisation and beliefs that parents, teachers, senior management teams and the education authorities have concerning the object of their demands. The psychologist must therefore know their prior ideas, implicit theories and beliefs, and what they think about the consultation process and its goals, and the elements that characterize the consultant's role.

How they intervene based on the model. At the pre-primary stage, the psychologists' work steers clear of approaches centring on the child's problems and difficulties, and focuses instead on supporting all the elements that define the system -the family and the school- in which the child learns and develops, considering support to the family not only as an indirect way of influencing the child's learning, but as a goal in itself. Psychologists must stimulate parents to participate in the diagnosis of the problem and in its solution, encouraging them to manifest their interests and wishes, and taking their opinions and preferences into account when setting the goals of the intervention. This involves relinquishing the role of expert in order to share the aims and outcomes of the intervention with the parents, which means taking decisions jointly as a basic principle of working in collaboration.

We use the work of Wesley and Buysse (2004) as our guide in explaining some of the elements defining the interaction between psychologist and educators during the different stages of consultation. It begins in the initial stage with the establishment of a relationship of mutual trust in which both parties' previous experiences are taken into account and the expertise each one can contribute to the process is examined. The next stage is the identification of the object of the intervention. Here it is essential to generate opportunities for discussing and sharing views on what is to be changed or improved and the elements that may influence this. This is followed by the choice of intervention strategies and their subsequent implementation, during which time the psychologist must put their skills and expertise at the service of the educa- tors. This means the psychologist's role in this stage must adapt to the on-going process, moving from that of observer to that of shaper or support professional.

Lastly, conclusions are offered as an invitation to reflect on issues such as how to ensure good-quality early education, what training psychologists working in this field should receive, what the intervention model should be and on what basis the model should be implemented - preventive or remedial.

\section{Referencias}

Anteproyecto de Marco Curricular para la Educación Infantil (1986). Ministerio de Educación y Ciencia: Madrid.

American Psychological Association. Council of Representatives (2006).Council of representatives policy statement on licensure. Washington, DC: American Psychological Association.

Blue-Banning, M., Summers, J. A., Frankland, H., Nelson, 1. y Beegle, G. (2004). Dimensions of family and professional partnerships: constructive guidelines for collaboration. Council for Exceptional Children, 70(2), 167-184.

Brazelton, T. B., y Greespan, S. I. (2005). Las necesidades básicas de la infancia. Barcelona: Graó.

Cox, D. D.(2005).Evidence-based interventions using homeschool collaboration. School Psychology Quarterly, 20, 473-497.

Del Río, C. (2009). La docencia de la ética profesional en los estudios de psicología en España. Papeles del Psicólogo, 30, 210-219.

Dunst, C. J. (2004). Revisiting Rethinking Early Intervention. En M. A. Feldman (Ed.), Early intervention. The essentials readings. (pp. 262-283). EE.UU: Blackwell Publishin.

Farrell P., Jimerson, S., Kalambouka, A. y Benoit, J. (2005). Teachers' perceptions of school psychologists in different countries. School Psychology International, 26, 525-544.

Farrell, P. (2009). El papel en desarrollo de los psicólogos escolares y educativos en el apoyo a niños, escuelas y familias. Papeles del Psicólogo, 30, 74-85.

Gine, C., Gracia, M., Vilaseca, R., y Balcells, A., (2009). Trabajar con las familias en atención temprana. Revista Interuniversitaria de Formación del Profesorado, 65 $(23,2)$ 95-113.

Grupo de Atención Temprana (G.A.T.) (2000). Libro Blanco de la AtenciónTemprana. Real Patronato de Prevención y de Atención a Personas con Minusvalía.

Grupo de Atención Temprana (G.A.T.) (2004): Recomendaciones Técnicas para el desarrollo de la Atención Temprana. Real Patronato sobre Discapacidad.

Hojnoski, R. L., y Missall, K. N. (2006). Addressing school readiness: Expanding school psychology in early education. School Psychology Review, 35, 602-614 .

Eurydice (2011), informe sistema educativo español 20092010. Recuperado de http://eacea.ec.europa.eu/educa- 
tion/eurydice/documents/eurybase/eurybase_full/reports/ ES_ES.pdf

Law, M., Hanna, S., King, G., Hurley, P., King, S., Kertoy, M (2003). Factors affecting family-centered service delivery for children with disabilities. Child: Care, Health and Development, 29, 357-366.

Ley 14/1970, de 4 de agosto, General de Educación y Financiamiento de la Reforma Educativa. Boletín Oficial del Estado Núm.187, de 6 de agosto (1970).

Ley Orgánica 1/1990, de 3 de diciembre, de Ordenación General del Sistema Educativo. Boletín Oficial del Estado Núm. 238, de 4 de octubre (1990).

Ley Orgánica 10/2002, de 23 de diciembre, de Calidad de la Educación. Boletín Oficial del Estado Núm. 307 de 24 de diciembre (2002).

Ley Orgánica 2/ 2006, de 3 de mayo, de Educación. Boletín Oficial del Estado Núm. 106 de 4 de mayo de (2006).

Méndez, L. y Escudero, I. (2011). El psicólogo educativo en EEUU y Australia. Psicología educativa, 17, 85-99.

Méndez, L (2011). El psicólogo educativo en España. Psicología educativa, 17, 39-56.

National Association of School Psychologists (2010). Model for Comprehensive and Integrated School Psychological Services. National Association of School Psychologists. Communique, 39(4).

National Association of School Psychologists. (2006). School psychology: A blueprint for training and practice (3rd. ed.). Bethesda: Author.

Nieto, J. M., y Portela, A. (2006). Una perspectiva ampliada sobre el asesoramiento en educación. Revista de educación, 339, 77-96.

Norwich, B. (2005). Future directions for professional Educational Psychology. School Psychology International Copyright, 26, 387-397.

Olaya, M. D. (1996). Los documentos del Plan Experimental de Educación Infantil. Revista Interuniversitaria de Formación del Profesorado, 25, 167-174.

Orden Ministerial, de 9 de diciembre de 1992, por la que se regulan la estructura y funciones de los Equipos de Orientación Educativa y Psicopedagógica. Boletín Oficial del Estado Núm. 303, de 18 de diciembre (1992).

Orden de 30 de abril de 1996, de la Dirección General de Renovación Pedagógica, por la que se dictan instrucciones sobre el funcionamiento de los equipos de orientación
Educativa y Psicopedagógica. Boletín Oficial del Estado Núm.20, de 13 de mayo (1996).

Orden de 14 de febrero de 1996, del Ministerio de Educación y Ciencia, por la que se regula el procedimiento para la realización de la evaluación psicopedagógica y el dictamen de escolarización de los alumnos con necesidades educativas especiales. Boletín Oficial del Estado Núm. 47, de 13 de febrero (1996).

Paniagua, G. (2011). Orientación y Asesoramiento en Educación Infantil. En Martín, Solé (coords.), Orientación Educativa, Modelos y estrategias de intervención. Vol. 1. (pp.187-2019). Barcelona: Graó.

Pozo, P., Sarría, E. y Méndez, L. (2006). Estrés en madres de personas con trastornos del espectro autista. Psicothema, $18,342-347$.

Ramey, C. T., Ramey, S. L. y Gaines, R. (2004). The transition to school: Building on preschool foundations and preparing for lifelong learning. En E. Zigler y S.J. Styfco (Eds.), The Head Start debates (pp. 397-413). Baltimore, MD: Brookes Publishing.

Real Decreto 1630/2006, de 29 de diciembre, por el que se establecen las enseñanzas mínimas del segundo ciclo de Educación Infantil. Boletín Oficial del Estado Núm. 4, de 4 de enero (2007).

Reschly, D. J. y Ysseldyke, J. E. (2002). Paradigm shift: The past is not the future. En A. Thomas y J. Grimes (Eds.), Best practices in school psychology. (pp.3-20). Bethesda, MD: National Association of School Psychologists.

Retortillo, F. y Puertas, E. (2004). Marco normativo de las necesidades especiales en las edades tempranas. Boletín del Real Patronato sobre Discapacidad, 57, 517.

Rosenfield, R., Levinsohn-Klyap, M., y Cramer, K. (2010). Educating consultants for practice in the schools. En García-Vázquez, E., Crespi, T., y Riccio, C (Eds). Handbook of education, training and supervision of school Psychologist. Vol. I (pp.259-278). NJ: Routledge.

Sarmiento, R. y Ruiz, E. (2011). Educación Temprana: desarrollo normativo autonómico 0-3 años. Revista Educación Inclusiva, 4 (1) 27-41.

Wesley, P., y Buysse, V. (2004). Consultation as a framework for productive collaboration in early intervention. Journal of Educational and Psychological Consulattion, 15(2), 127-150.

Manuscrito recibido: 25/03/2012

Revisión recibida: 09/10/2012

Manuscrito aceptado: 29/10/2012 